\title{
Coumarin-Chalcone Hybrids as new scaffolds in drug discovery
}

\author{
Saleta Vázquez Rodríguez ${ }^{\mathrm{a}}$, Silvia Serra ${ }^{\mathrm{a}}$, Lourdes Santana ${ }^{\mathrm{a}}$ and Eugenio \\ Uriarte $^{\mathrm{a}}$.
}

${ }^{a}$ Department of Organic Chemistry, Faculty of Pharmacy, University of Santiago de Compostela, 15782, Spain.

*E-mail: $\underline{\text { svre77@hotmail.com }}$

\begin{abstract}
The first hydroxilated series of coumarin-chalcone derivatives has been synthesize starting from the corresponding salicyl aldehyde and $\beta$-ketoester precursors by a Knoevenagel reaction in order to obtain the methoxy derivatives which have been further hydrolyzed with a Lewis acid.
\end{abstract}

Keywords: Coumarin, Chalcone, Knoevenagel

\section{Introduction}

Coumarins and chalcone are a family of natural and synthetic compounds that have raised an enormous interest for a long time due to its biological applications. One of the biggest applications of coumarins resides on the wide range of pharmacological activities such as antimicrobial, antiviral, antioxidant, antiplatelet, vasorelaxant or enzymatic inhibitors ${ }^{1,2,3,4}$ so this coumarin scaffold give us a hint in the discovery and development of new drugs. Besides coumarins, chalcones share a wide range of this biological properties like antiproliferative, cardioprotector, or anti-inflammatory activity ${ }^{5,6,7}$ and in the present year chalcones have been proposed as valid scaffolds for monoamino oxidases inhibitors ${ }^{8}$. 
On the other hand, many studies suggest that polyphenols may be involved in neurotoxic or cyclooxygenase activity as well as they play a role as chemopreventive and tumor growth inhibition agents and their protective properties against oxidative stress. $^{9,10,11}$ It is for these reasons that we have synthesized a novel series of hydroxylated coumarin-based compounds containing a chalcone moiety in order to make different biological essays and test their potential pharmacological activities.

\section{Results and Discussion}

The aim of this work has been to synthesize new hydroxy coumarin-chalcone hybrids which contain the hydroxyl sustituents in the aromatic rings that can potentially be used as new lead compounds in drug discovery.

Compounds were synthesized using a two steps synthetic strategy that allows us to obtain methoxy derivatives which were further hydrolyzed using a Lewis acid. (Figure 1)

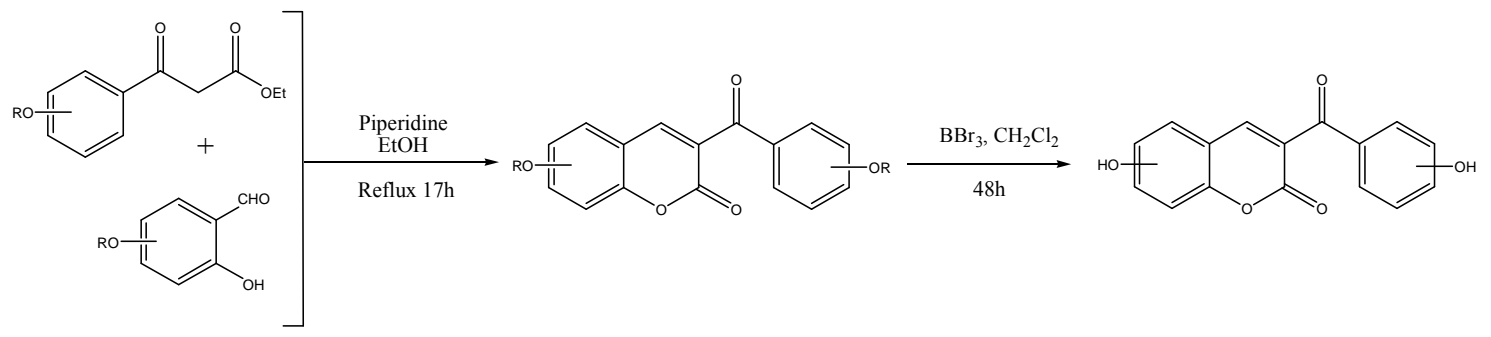

Figure 1. Synthetic route used to prepare hydroxy coumarin-chalcone hybrids.

The main scaffold with methoxy substituents was prepared by a Knoevenagel reaction in basic conditions in $82-93 \%$ yields. First synthesized derivatives have the methoxy sustituens placed in positions 8 and 4', (Table 1)

Table 1. Coumarin-chalcone hybrids

\begin{tabular}{|c|c|c|c|c|}
\hline Compound & $\mathbf{1}$ & $\mathbf{2}$ & $\mathbf{3}$ & $\mathbf{4}$ \\
\hline $\mathbf{R}_{\mathbf{1}}$ & $-\mathrm{H}$ & $-\mathrm{Br}$ & $-\mathrm{H}$ & $-\mathrm{Br}$ \\
\hline
\end{tabular}




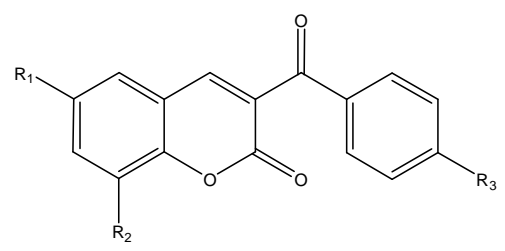

\begin{tabular}{|c|c|c|c|c|}
\hline $\mathbf{R}_{\mathbf{2}}$ & $-\mathrm{H}$ & $-\mathrm{OMe}$ & $-\mathrm{H}$ & $-\mathrm{OH}$ \\
\hline $\mathbf{R}_{\mathbf{3}}$ & $-\mathrm{OMe}$ & $-\mathrm{H}$ & $-\mathrm{OH}$ & $-\mathrm{H}$ \\
\hline
\end{tabular}

Hydroxy derivatives were further prepared from the ether precursors by catalysis with a Lewis acid, $\mathrm{BBr}_{3}$ in $\mathrm{CH}_{2} \mathrm{Cl}_{2}{ }^{12}$. Purification of compounds $\mathbf{1 - 2}$ was made by recristalization in $\mathrm{MeOH}$, while compounds 3-4 were purified by flash chromatography.

The followed methodologies to prepare compounds $\mathbf{1 - 2}$, in a parallel synthesis way, bring the opportunity of synthesize structural related compounds with punctual modifications in the aromatic rings depending on the starting materials.

Biological assays as vasodilatators, antioxidants and monoaminooxidase inhibitors are currently being carried out and results will be further presented.

\section{General Experimental Procedure}

All reactions were carried out under dry and deoxygenated argon atmosphere. Solvents were used as anhydrous by reflux of each solvent over an appropriate dryer agent and further distillate under argon atmosphere.

Qualitative identification of the compounds and course of the reactions were visualized using TLC plates (Merck, silica gel $60 \mathrm{~F}_{254}$ ) under UV light (254-366 nm). Melting points were determined using a Reichert Kofler thermopan or in capillary tubes on a Büchi 510 apparatus and are uncorrected. ${ }^{1} \mathrm{H}$ NMR spectra were recorded on a Bruker WM-250 at $250 \mathrm{MHz}$ using TMS as internal standard (chemical shifts in $\delta$ values, $J$ in $\mathrm{Hz}$ ).

General procedure for the preparation of 3-benzoylcoumarins: In a round bottom flask the appropiated salicylaldehyde (1 equiv.unless otherwise noted) and ethyl benzoylacetate ( 1 eq. unless otherwise indicated) were dissolved in dry EtOH. Were then added 5 drops of piperidine, and the reaction was subjected to reflux. The reaction was monitored by TLC and after the end of it, the solution was cooled on ice and the precipitate was filtered and washed with ether. The solid obtained was purified by recrystallization in $\mathrm{MeOH}$ : $\mathrm{CH} 2 \mathrm{Cl} 2$ and / or column chromatography. 
General procedure for the preparation of hydroxy 3-benzoylcoumarins: Ether derivatives of 3-benzoylcoumarins (1 eq.) were dissolved in dry $\mathrm{DCM}$ and $\mathrm{BBr}_{3}$ (20 eq.) was added dropwise at room temperature in a sealed tube. Mixture was stired and heated at $80^{\circ} \mathrm{C}$ for $48 \mathrm{~h}$. After cooling to room temperature, the crude reaction mixture was quenched carefully with ice, water and $1 \mathrm{~N} \mathrm{HCl}$. The aqueous layer was extracted with AcOEt $(3 \times 50 \mathrm{~cm} 3)$ and the combined organic extracts were washed with saturated aqueous $\mathrm{NaHCO} 3$ and brine, dried $\left(\mathrm{MgSO}_{4}\right)$ and concentrated to dryness. 3-(p-methoxybenzoyl)coumarin (1): Yield: 93\%. ${ }^{1} \mathrm{H}-\mathrm{NMR}\left(250 \mathrm{MHz}, \mathrm{DMSO}-d_{6}\right) \delta$ ppm 8.34 (s, 1H, H-4), 7.92 (d, $J=8.8$ Hz, 2H, H-2', H-6'), 7.83 (d, J=8.6 Hz, 1H, H5), 7.70 (d, $J=7.26 \mathrm{~Hz}, 1 \mathrm{H}, \mathrm{H}-8), 7.55-7.33$ (m, 2H, H-6, H-7), 7.05 (d, $J=8.8 \mathrm{~Hz}$, 2H, H-3', H-5'), 3.85 (s, 3H, - $\left.\mathrm{CH}_{3}\right)$. MS m/z (\%):281 ([M+1] $\left.]^{+}, 19\right), 280$ ([M] $\left.]^{+}, 82\right), 135$ (100), 77 (20).

3-benzoyl-5-bromo-8-methoxycoumarin (2): Yield: 82\%. ${ }^{1} \mathrm{H}-\mathrm{NMR}(250 \mathrm{MHz}$, $\left.\mathrm{CDCl}_{3}\right) \delta \mathrm{ppm} 7.78$ (s, 1H, H-4), 7.69 (d, J=7.3, 2H, H-2', H-6'), 7.51-7.24 (m, 3H, H3', H-4', H-5'), 7.14 (s, 1H, H-5), 7.09 (s, 1H, H-7), 3.82 (s, 3H, - $\mathrm{CH}_{3}$ ) MS m/z (\%):360 $\left([\mathrm{M}+1]^{+}, 18\right), 359\left([\mathrm{M}]^{+}, 58\right), 358(18), 357$ (58), 105 (100), 77 (70).

3-(p-hydroxybenzoyl)coumarin (3): Yield: $67 \%$. ${ }^{1} \mathrm{H}$ NMR: $\left(250 \mathrm{MHz}, D M S O-d_{6}\right) \delta$ ppm 10.6 (s, 1H, -OH), 8.3 (s, 1H, -H4), 7.8 (d, J=8.5 Hz, 4H, -H2', -H3', -H5', -H6'), 7.7 (t, $J=7.50 \mathrm{~Hz}, 1 \mathrm{H},-\mathrm{H} 5), 7.5-7.3$ (m, 2H, H6, H7), 6.9 (d, $J=8.5 \mathrm{~Hz}, 1 \mathrm{H},-\mathrm{H} 8)$. MS $m / z 267\left([\mathrm{M}+1]^{+}, 12\right), 266\left([\mathrm{M}]^{+}, 57\right), 249(22)$

3-benzoyl-5-bromo-8-hydroxycoumarin (4): Yield: 52\%. ${ }^{1} \mathrm{H}$ NMR: $(250 \mathrm{MHz}$, DMSO-d $\left.{ }_{6}\right) \delta$ ppm 10.8 (s, 1H, -OH), 7.8 (d, $J=7.9$ Hz, 2H, H2', H6') 7.6-7.3 (m, 3H, H3', -H4', -H5'), 7.0 (s, 1H, -H5), 6.8 (s, 1H, H7). MS m/z 346 ([M+1] $\left.]^{+}, 18\right), 345$ $\left([\mathrm{M}]^{+}, 62\right), 344(18), 343(53), 105$ (100).

\section{References}

${ }^{1}$ Borges, F.; Roleira, F.; Santana, L.; Uriare, E., Curr. Med. Chem., 2005, 12, 887-916

${ }^{2}$ Orallo, F.; Alvarez, E.; Camiña, M. et all, Mol. Pharmacol., 2002, 61, 294-302

${ }^{3}$ Leiro, J.; Alvarez, E.; Arranz, J.A.; Laguna, R.; Uriarte, E. Y Orallo, F., J. Leukoc.

Biol., 2004, 75, 1156-1165

${ }^{4}$ Santana, L.; Uriarte, E.; González-Díaz, H.; Zagotto,G.; Soto-Otero, R.; E. MéndezÁlvarez, E. J. Med. Chem., 2006, 49, 1118. 
${ }^{5}$ Cabrera, M.; Simoens, M.; Falchi, G.; Lavaggi, M. L.; Piro, O. E.; Castellano, E.E.; Vidal, A.; Azqueta, A.; Monge, A.; Lopez de Cerain, A.; Gonzalez, M. Bioorg. Med. Chem., 2007, 15(10), 3356-3367.

${ }^{6}$ Dong, X.; Liu, T.; Yan, J.; Wu, P.; Chen, J.; Hu, Y., Bioorg. Med. Chem., 2009, 17(2), 716-726.

${ }^{7}$ Nowakowska, Z. Eur J. Med. Chem., 2007, 42(2), 125-37.

${ }^{8}$ Chimenti, F.; Fioravanti, R.; Bolasco, A.; Chimenti, P.; Secci, D.; Rossi, F.; Yanez, M.; Orallo, F.; Ortuso, F.; Alcaro, S., J. Med.Chem., 2009, 52(9), 2818-2824.

${ }^{9}$ Lodovici, M.; Menichetti, S.; Viglianisi, C.; Caldini, S.; Giuliani, E., Bioorg. Med. Chem. Lett., 2006, 16(7), 1957-1960

${ }^{10}$ Rocha-Gonzalez, H.I.; Ambriz-Tututi, M.; Granados-Soto, V., CNS Neuroscience \& Therapeutics, 2008, 14(3), 234-247

${ }^{11}$ Shinichi Kitada, M.L., Sina Sareth, D.; Zhai, J. C. Reed, and Pellecchia M., J. Med. Chem., 2003, 46 (20), pp 4259-4264

${ }^{12}$ Martín Santamaría, S.; Rodríguez, J. J.; de Pascual-Teresa, S.; et all., Org. Biomol. Chem., 2008, 6, 3486-3492 